ISSN 0258-7122 (Print), 2408-8293 (Online)

Bangladesh J. Agril. Res. 42(1): 53-65, March 2017

\title{
DYNAMICS OF PHYTOHORMONES AND THEIR RELATIONSHIP WITH CHALKINESS OF EARLY INDICA RICE UNDER DIFFERENT POST-ANTHESIS TEMPERATURE REGIMES
}

\author{
MOHAMMED HUMAYUN KABIR ${ }^{1}$, QING LIU ${ }^{2}$, YI SU ${ }^{3}$ \\ ZHIGANG HUANG ${ }^{4}$ AND LANGTAO XIAO ${ }^{5}$
}

\begin{abstract}
A pot experiment on an early indica rice cv. 'Shenyou9576' was conducted in the net house of Hunan Agricultural University, Changsha, Hunan, PR China during the early growing season of 2013 to investigate the influence of varying temperatures on chalkiness rate, head rice rate, and phytohormones, namely indole-3-acetic acid (IAA), gibberellins $\left(\mathrm{GA}_{1}\right.$ and $\left.\mathrm{GA}_{4}\right)$, zeatin $(\mathrm{Z})$, zeatin riboside (ZR) and abscisic acid (ABA) both in flag leaves and grain endosperm during grain filling period. The treatments comprised three temperature regimes which are designated as the high $\left(35 / 28^{\circ} \mathrm{C}\right.$ - day/night $)$, low $\left(25 / 20^{\circ} \mathrm{C}\right.$ - day/night $)$ and natural condition as the control $\left(35 / 25^{\circ} \mathrm{C}\right.$ - day/night). The results showed that the maximum chalkiness rate was $61.11 \%$ under high temperature and the minimum $(22.59 \%)$ under low temperature. The lowest head rice rate was $42.76 \%$ under high temperature followed by $49.91 \%$ in the control, while the highest $(62.33 \%)$ under low temperature. The contents of $\mathrm{GA}_{1}, \mathrm{GA}_{4}, \mathrm{Z}$ and $\mathrm{ZR}$ were decreased gradually from 7 to 35 days after anthesis (DAA) irrespective of treatments. IAA content began to decrease from 14 DAA and continued up to 35 DAA and ABA was reduced from 28 to 35 DAA under low temperature in comparison to control and high temperature. ABA content was increased from 7 to $21 \mathrm{DAA}$ and then declined at high temperature. The results showed that contents of $\mathrm{GA}_{1}, \mathrm{GA}_{4}, \mathrm{Z}, \mathrm{ZR}$ were high at low temperature compared to high temperature and control. IAA content was also high during grain development period at low temperature except 7 DAA. Higher phytohormone contents were observed in endosperm than in flag leaves. Phytohormone content ratio (endosperm: flag leaves) was found highest in IAA and the lowest in $\mathrm{GA}_{1}$. A significant positive correlation was found between ABA and chalkiness rate during early to mid grain filling period, while significant negative correlations were noticed between chalkiness rate and other phytohormones during grain filling period. Correlation results revealed that increased level of ABA during early to mid grain filing period caused by high temperature was more responsible for development of chalkiness.
\end{abstract}

Keywords: Rice (Oryza sativa L.), high temperature, phytohormones, head rice rate, chalkiness rate.

${ }^{1}$ Senior Scientific Officer, Adaptive Research Division, Bangladesh Rice Research Institute (BRRI), Gazipur-1701, Bangladesh, ${ }^{2-5}$ Hunan Provincial Key Laboratory of Phytohormones and Growth Development, Hunan Agricultural University and Hunan Provincial Key Laboratory for Crop Germplasm Innovation \& Utilization, Changsha 410128, Hunan, China. 


\section{Introduction}

Rice (Oryza sativa L.) is one of the most important food crops in the world as it serves as the staple food of more than a half of the world's population. Grain quality is one of the most important factors in rice production and in economic returns for the farmers. The most important rice quality components include milling, appearance, cooking, eating and nutrient qualities (Han et al., 2004; Koutroubas et al., 2004). Appearance quality of rice grain depends on endosperm color and grain dimension, while endosperm color is mainly determined by the degree of chalkiness i.e. the white opaque part in rice endosperm. The appearance quality of rice kernel with more chalkiness influences on the milling percentage, cooking, nutritional and eating quality. Rice grains with more chalkiness are likely to break during milling, which affects the commercial value of milled rice and the market acceptability (Borrell et al., 1997). Chalkiness forms longitudinal and transverse cracks, and causes broken or loosen rice grain when cooked, resulting in poor eating quality. Previous studies showed that the environmental (specially daily average-temperature during grain filling) and genetic factors are associated with chalkiness formation (Dong et al., 2006; Koutroubas et al., 2004; Zhang et al., 2008). It is also reported that high temperature stress affects the occurrence of chalky kernels (Patindol and Wang, 2003).

During the grain filling period, rice grains act as strong carbohydrate sinks (Cao et al., 1992). It is assumed that hormones in a sink organ are prominent factors in determining sink strength and it has been proposed that grain development may be mediated through endogenous phytohormones (Naik and Mohapatra, 1999; Yang et al., 2000, 2001). It is generally believed that plant hormones including indole-3-acetic acid (IAA), abscisic acid (ABA), zeatin riboside (ZR), and gibberellin (GA) are closely associated with grain development (Wobus and Weber, 1999). The intrinsic factors are responsible for variations in grain development as well as chalkiness. The limited work on the characteristics of endogenous phytohormones changes and their relationships with rice grain chalkiness under post-anthesis temperature conditions was done. Therefore, the present study was conducted to investigate the influence of different temperatures on chalkiness, head rice rate, and changes of IAA, gibberellins $\left(\mathrm{GA}_{1}\right.$ and $\left.\mathrm{GA}_{4}\right)$, cytokinins ( $\mathrm{Z}$ and $\mathrm{ZR}$ ) and $\mathrm{ABA}$ contents throughout the grain filling period and to reveal the relationship between chalkiness and the phytohormone content.

\section{Materials and Method}

\section{Experiment description}

The experiment was carried out in the net house of Hunan Agricultural University, Changsha, Hunan, PR China, in the early rice growing season from 
April to August of 2013. The tested rice variety was a heat tolerant early indica rice 'Shenyou 9576'. Germinated seeds of rice were pre-grown with complete Kimura B nutrient solution (Yoshida et al., 1976) in a green house until 15 days. Seedlings were then transferred to earthen pots of $30 \mathrm{~cm}$ in diameter and $32 \mathrm{~cm}$ in depth filled with $7.0 \mathrm{~kg}$ of sieved, dry paddy soil (the contents of soil organic matter, alkaline hydrolytic nitrogen, effective phosphorus, available potassium were $1.8 \%, 66.2 \mathrm{mg} \mathrm{kg}^{-1}, 8.5 \mathrm{mg} \mathrm{kg}^{-1}$, and $8.0 \mathrm{mg} \mathrm{kg}^{-1}$, respectively, and soil $\mathrm{pH}$ was 5.4) amended with $1.0 \mathrm{~g} \mathrm{CO}\left(\mathrm{NH}_{2}\right)_{2}, 0.4 \mathrm{~g} \mathrm{P}_{2} \mathrm{O}_{5}$, and $0.6 \mathrm{~g} \mathrm{~K}_{2} \mathrm{O}$ per kg soil to grow. Two seedlings were transplanted in each earthen pot. Proper management practices were provided as per requirement for proper growth. The treatment consisted of three temperature regimes which are designated as high temperature $\left(35 / 28^{\circ} \mathrm{C}\right.$, $12 \mathrm{~h}$ light $/ 12 \mathrm{~h}$ dark, $75-80 \%$ relative humidity), low temperature $\left(25 / 20^{\circ} \mathrm{C}, 12 \mathrm{~h}\right.$ light $/ 12 \mathrm{~h}$ dark, $75-80 \%$ relative humidity) and natural condition $\left(35 / 25^{\circ} \mathrm{C}\right.$ - day/night) as control. The treatments were imposed after anthesis by transferring pots into different growth chambers, but for the control treatment pots were kept in the net house under natural condition. The experiment was performed according to a complete randomized design (CRD) with three replications.

\section{Sampling method}

Panicles and flag leaves from each treatment were collected at 7-day interval after anthesis i.e. 7, 14, 21, 28, 35 days after anthesis (DAA). Samples were collected on 9.00 to 11.00 am and immediately wrapped in aluminum foil and frozen in liquid nitrogen, then placed into a sealed plastic bag and stored at $-60^{\circ} \mathrm{C}$ until use for different analysis. Rice grains were harvested at $35 \mathrm{DAA}$ and then were sun dried to achieve $14 \%$ moisture content. Rough rice (paddy rice) was dehusked by a SBS- 80 dehuller, then was polished by a rice polisher for 2 minutes. Milled rice samples were kept in sealed bags under refrigeration $\left(4^{\circ} \mathrm{C}\right)$ for later analysis.

\section{Chalkiness and head rice rate measurement}

Chalkiness was measured with a system composed of a scanner and a special software Chalkiness 2.0 developed by Hunan Agricultural University (Chen et al., 2011). Head rice refers to the whole grains of milled rice and was computed by using the following equation (Gummert, 2010).

$$
\text { Head rice }(\%)=\frac{\text { Wt of whole grains }}{\text { Wt of paddy samples }} \times 100
$$

Extraction, purification and determination of indole-3-acetic acid (IAA), gibberellins $\left(\mathrm{GA}_{1}\right.$ and $\left.\mathrm{GA}_{4}\right)$, zeatin $(\mathrm{Z})$, zeatin riboside $(\mathrm{ZR})$ and abscisic acid (ABA) 
Fresh plant materials (panicles or flag leaves) of 500 to $700 \mathrm{mg}$ were frozen in liquid nitrogen and ground in a mortar with a pestle. After adding $800 \mu \mathrm{L}$ of $80 \%$ methanol, samples were mixed thoroughly and kept overnight at $4{ }^{\circ} \mathrm{C}$. Then the mixtures were centrifuged at $4,800 \mathrm{~g}$ and $4^{\circ} \mathrm{C}$ for $10 \mathrm{~min}$. The supernatant was transferred to a new tube and the residues were re-extracted with $800 \mu \mathrm{L}$ of $80 \%$ methanol for 4 hours at $4{ }^{\circ} \mathrm{C}$ for two times. The supernatants were combined and dried in a Jouan RCT-60 vacuum concentration system, then dissolved in $200 \mu \mathrm{L}$ of $0.1 \mathrm{molL}^{-1}$ buffer ( $\mathrm{pH} 7.8$ ). The aqueous phase was purified through a Waters Sep-pak $\mathrm{C}_{18}$ cartridge (Waters, USA) and followed by a wash with $800 \mu \mathrm{L}$ of $\mathrm{ddH}_{2} \mathrm{O}$, and then the eluted was vacuum freeze dried. The dried extract was dissolved in $40 \mu \mathrm{L}$ of $50 \%$ methanol and used for an assay by a SHIMADJU LCMS-8030 mass spectrometer. A BEH C 18 column $(100 \mathrm{~mm} \times 2.1 \mathrm{~mm}, 1.7 \mu \mathrm{m})$ was used and mass-to-charge ratio were 174/130.05, 220/136.1, 352.2/220.1, $347 / 259.2,331 / 243.25,263 / 153.2$ for IAA, Z, ZR, GA,$G_{4}$ and ABA, respectively.

\section{Statistical analysis}

All experimental data were analyzed following analysis of variance. Mean separation of the treatments was done by using Least Significant Difference (LSD) at 5\% level. All statistical analysis was performed by using a statistical software, DPS version 12.01 and Microsoft Excel 2003 (Microsoft, USA) was used to generate graphs.

Under the natural condition (control) of the experiment site, daily maximum, minimum and average temperature $\left({ }^{\circ} \mathrm{C}\right)$ from flowering to harvest are shown in Fig. 1 . The average daily temperature lies within $25^{\circ} \mathrm{C}$ to $35^{\circ} \mathrm{C}$ during this period.

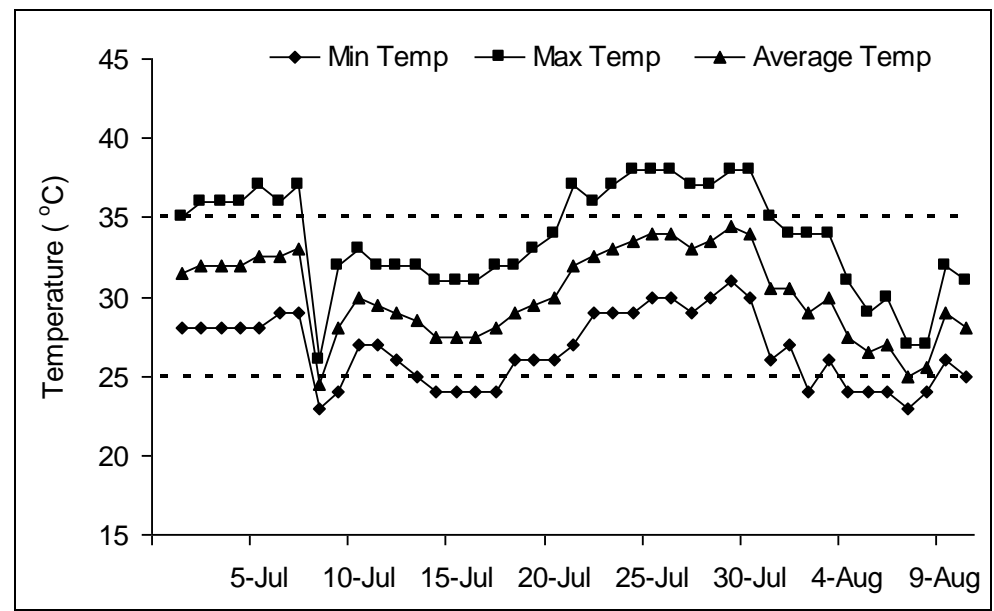

Fig. 1. Daily maximum, minimum and average air temperature $\left({ }^{\circ} \mathrm{C}\right)$ during anthesis to maturity at Changsha, Hunan, China. 


\section{Results and Discussion}

\section{A. Results}

Changes of chalkiness rate and head rice rate under different temperature regimes:

Chalkiness $(\%)$ in different temperature treatments were significantly affected by different temperature regimes (Fig. 2a). The highest chalkiness rate $(61.11 \%$ ) was observed under high temperature and the lowest chalkiness $(22.59 \%)$ under low temperature, where in control, chalkiness rate was in between high and low temperature, which was $47.81 \%$. Therefore, chalkiness (\%) was increased by high temperature stress at the grain filling stage. The highest head rice was $62.33 \%$ under low temperature followed by $49.91 \%$ under the control and the lowest $(42.76 \%)$ under high temperature (Fig. 2b). Therefore, the results indicate that high temperature stress has a negative effect on head rice (\%), while low temperature has a positive effect.

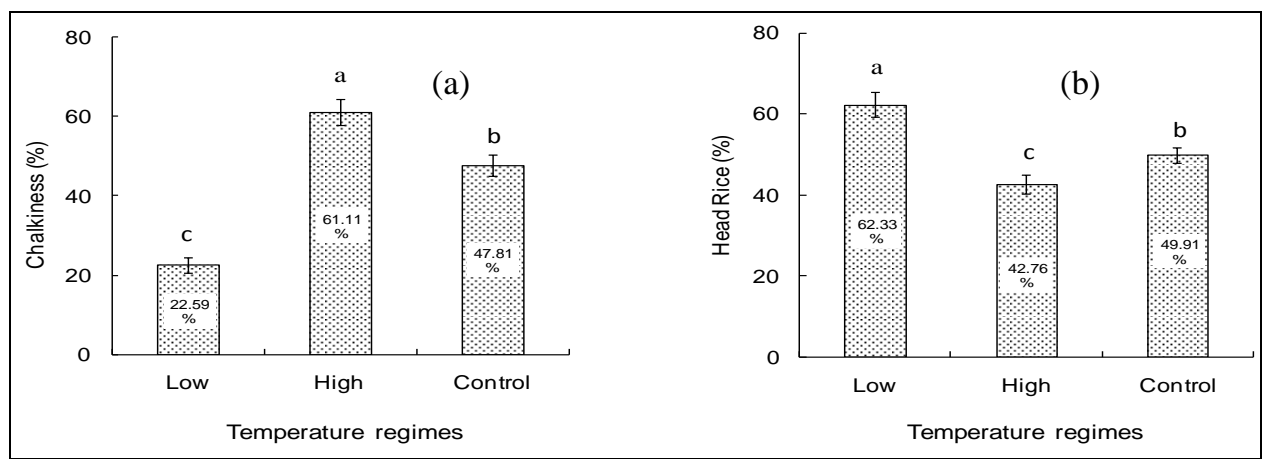

Fig. 2. (a) Chalkiness (\%) and (b) head rice (\%) influenced by temperature regimes after anthesis. Vertical bars represent $\pm \mathrm{SE}$ of the mean $(n=3)$. Bars with different letters are significantly different at $5 \%$ level by LSD.

\section{Changes of IAA content in rice flag leaf and endosperm:}

The variation of IAA content both in flag leaf and endosperm of rice was significant among the treatments in all of the studied DAAs (Fig. 3a, b). The highest IAA content (43.39 $\mathrm{ngg}^{-1} \mathrm{FW}$ ) was observed under high temperature at the early grain filling stage in flag leaf, and then a decreasing trend was observed up to 35 DAA. At the middle grain filling stage (14 DAA) IAA content in flag leaf was the highest under low temperature followed by the control and high temperature treatment. In rice endosperm, at the early grain filling period the highest IAA content $\left(663.58 \mathrm{ngg}^{-1} \mathrm{FW}\right)$ was observed under high temperature treatment followed by the control $\left(630.24 \mathrm{ngg}^{-1} \mathrm{FW}\right)$ and low temperature treatment (576.49 $\mathrm{ngg}^{-1} \mathrm{FW}$ ), while mid to the late grain filling period, the highest IAA content was recorded under low temperature. Therefore, low temperature is favorable for higher IAA content at the middle and the later grain 
filling period. Furthermore, irrespective of temperature treatments, a decreasing trend of IAA was observed from 14 to 35 DAA.
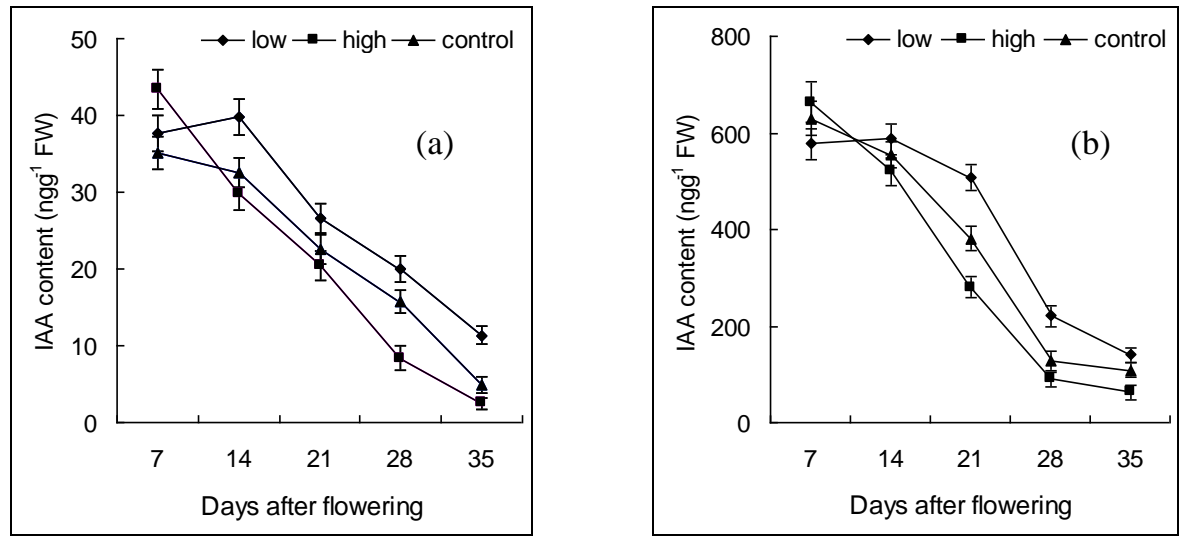

Fig. 3. Changes of IAA content in (a) rice flag leaves and (b) rice endosperm after anthesis under different temperature regimes. Vertical bars represent $\pm \mathrm{SE}$ of the mean $(n=3)$.
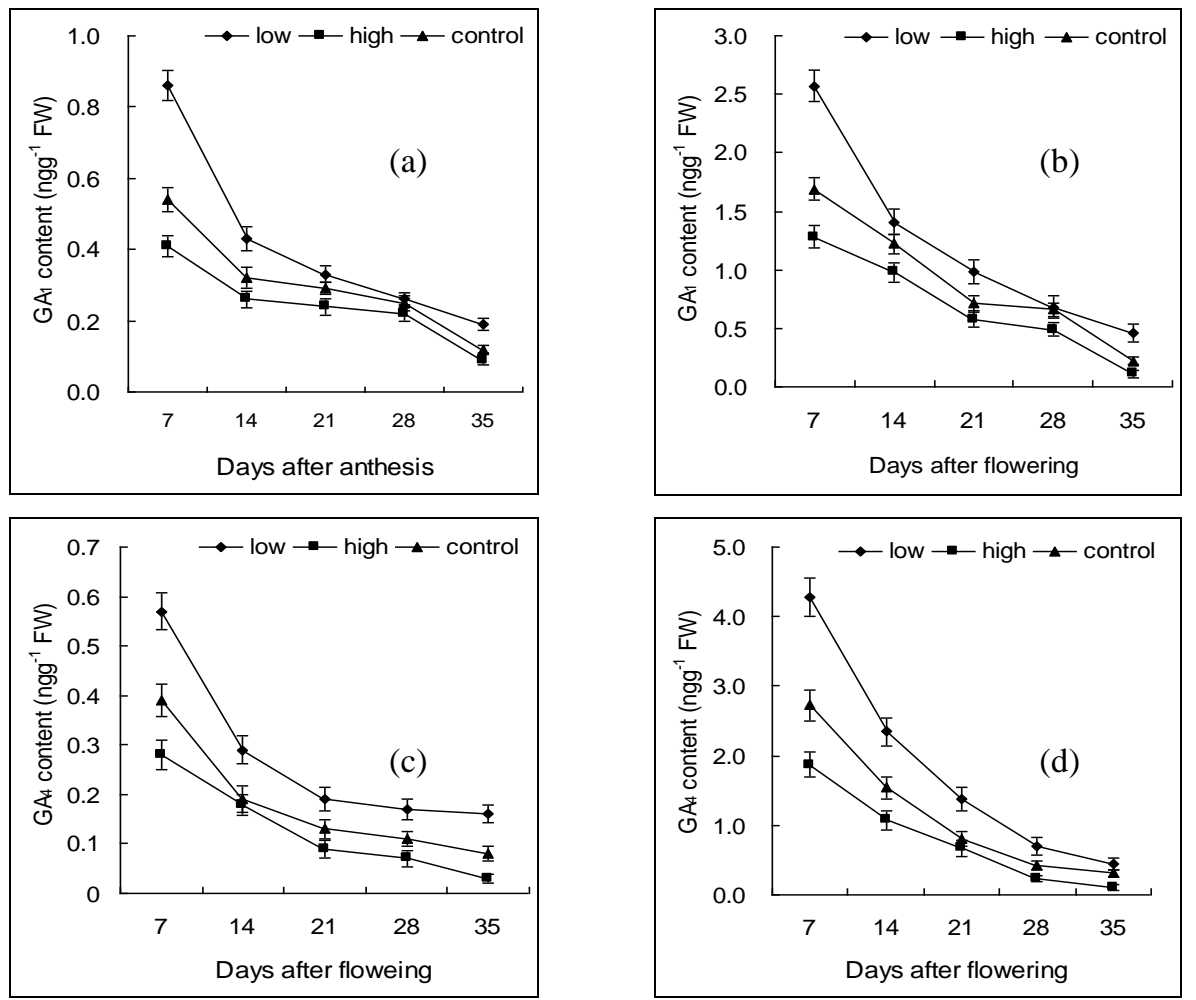

Fig. 4. Changes of GA 1 content in (a) rice flag leaves, (b) rice endosperm and $\mathrm{GA}_{4}$ content in (c) rice flag leaves, (d) rice endosperm after anthesis under different temperature regimes. Bars represent $\pm S E$ of the mean $(n=3)$. 
Changes of gibberelins $\left(\mathrm{GA}_{1}\right.$ and $\left.\mathrm{GA}_{4}\right)$ contents in rice flag leaf and endosperm:

The effect of temperature on $\mathrm{GA}_{1}$ and $\mathrm{GA}_{4}$ content in rice flag leaf and endosperm displayed a marked variation at all the studied DAAs (Figs. 4a, b, c, d). Irrespective of the temperature treatments higher $\mathrm{GA}_{1}$ and $\mathrm{GA}_{4}$ content were observed at the early grain filling period, where the lowest under high temperature and the highest $\mathrm{GA}_{1}$ and $\mathrm{GA}_{4}$ content under low temperature throughout the grain filling period both in rice flag leaf and grain. $\mathrm{GA}_{1}$ content in rice endosperm under low temperature was 1.52 fold and 2 fold higher, while $\mathrm{GA}_{4}$ content under low temperature increased 1.56 fold and 2.28 fold than the control and high temperature, respectively.
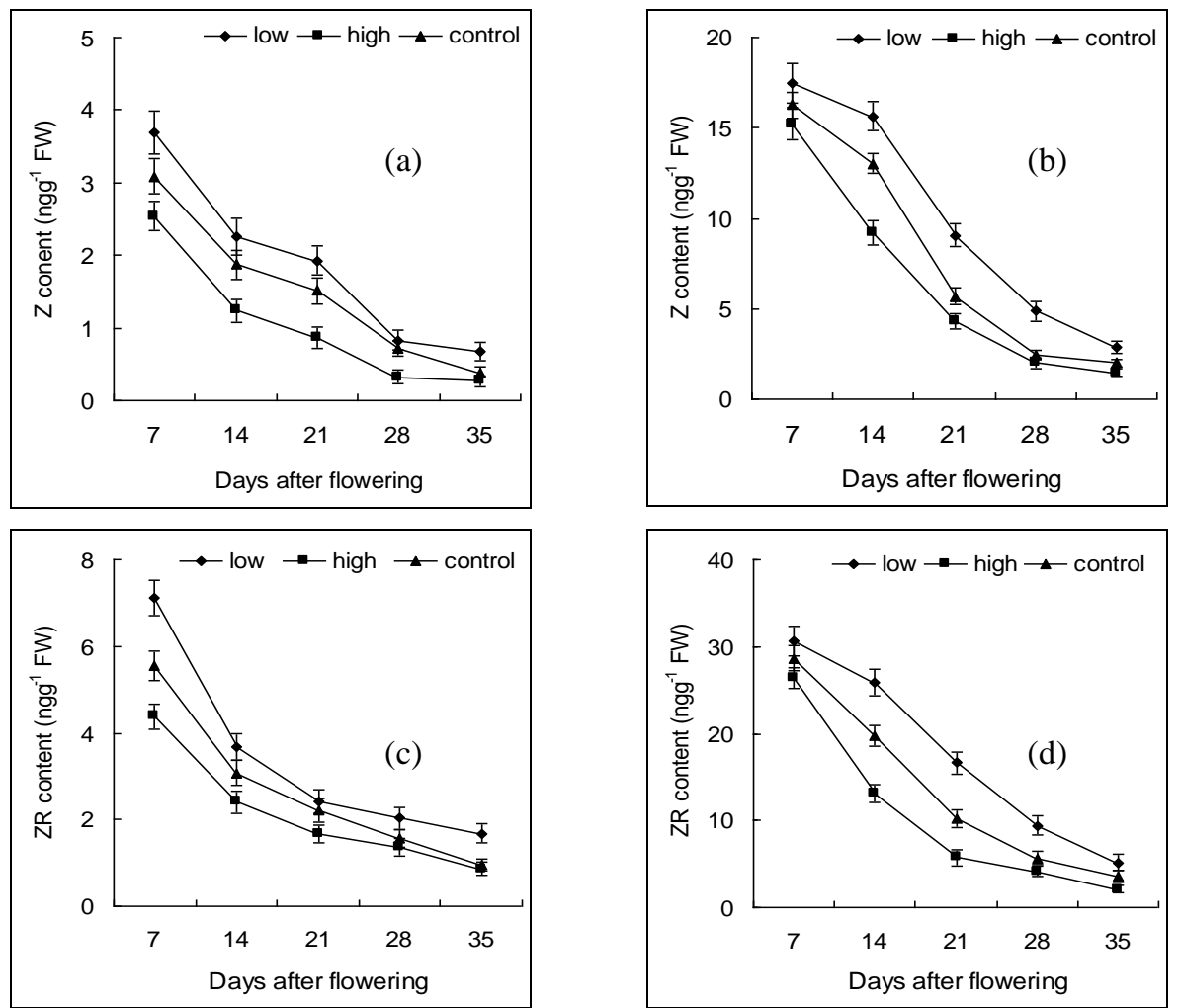

Fig. 5. Changes of $Z$ content in (a) rice flag leaves, (b) rice endosperm and $Z R$ content in (c) rice flag leaves, (d) rice endosperm after anthesis under different temperature regimes. Bars represent $\pm S E$ of the mean $(n=3)$.

\section{Changes of cytokinins ( $Z$ and $Z R$ ) contents in rice flag leaf and endosperm:}

The magnitude of the differences among the three temperature regimes on $\mathrm{Z}$ and $\mathrm{ZR}$ accumulation in rice flag leaf and endosperm were important ones (Figs. 5a, b, c, d). High temperature displayed the lowest $\mathrm{Z}$ and $\mathrm{ZR}$ contents, while low 
temperature showed the highest $\mathrm{Z}$ and $\mathrm{ZR}$ contents both in rice flag leaf and kernel throughout the grain filling period. On an average higher $\mathrm{Z}$ and $\mathrm{ZR}$ contents were found at the early grain filling period than at the middle or later grain filling period. At 7 DAA, $\mathrm{Z}$ content in rice endosperm under low temperature was $7.44 \%$ and $14.71 \%$ higher than the control and high temperature, respectively. However, $\mathrm{Z}$ content in rice endosperm was about 6 times higher than flag leaf. Relative to the control, exposure to high temperature ZR accumulation in kernel was reduced by $8-44 \%$ and increased by $7-65 \%$ in the different DAAs. The results showed that there was more depletion of $\mathrm{Z}$ and $\mathrm{ZR}$ contents under high temperature and the control than low temperature both in rice flag leaf and endosperm.

\section{Changes of abscisic acid (ABA) level in rice flag leaf and endosperm:}

Significant effect was found among the temperature treatments on ABA content both in rice flag leaf and endosperm (Figs. 6a, b). Under high temperature ABA content in flag leaf increased gradually from 7 DAA, and had a peak $\left(26.45 \mathrm{ngg}^{-1}\right.$ FW) at 21 DAA, while under low temperature reached a peak value of $26.77 \mathrm{ngg}$ ${ }^{1} \mathrm{FW}$ at 28 DAA. High temperature treatment showed a significantly higher ABA level in rice endosperm compared to control and peaked at 21 DAA (Fig. 6b). Low temperature treatment showed significantly lower ABA level up to 21 DAA, but on wards to 28 and 35 DAA in rice endosperm showed higher ABA level.
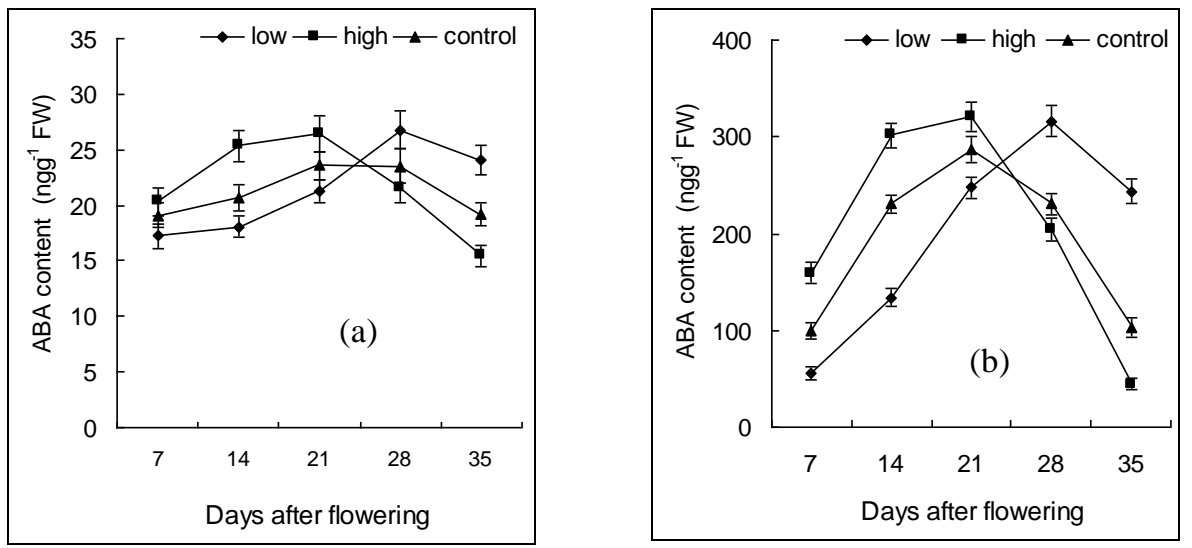

Fig. 6. Changes of ABA content in (a) rice flag leaves (b) and rice endosperm after anthesis under different temperature regimes. Bars represent $\pm \mathrm{SE}$ of the mean $(n=3)$.

\section{Comparison of phytohormones content between developing endosperm and flag leaves of rice:}

The influence of phytohormones on the metabolism of carbohydrates in rice endosperm as well as in photosynthetic tissue, both in the developing endosperm 
and flag leaf of rice and the phytohormones content ratio between developing endosperm and flag leaves of rice are presented in Table 1. Several-folds (1.22 to 19.12 fold) higher phytohormones were observed in endosperm than in flag leaves. The highest ratio (8.56 to 19.12) was noted in IAA than others hormones irrespective of treatments followed by $\mathrm{ABA}$, while the ratio was lower for $\mathrm{GA}_{1}$ (1.22 to 3.77). However, the ratio between rice endosperm and flag leaves for $\mathrm{GA}_{4}$ was markedly higher than $\mathrm{GA}_{1}$. The ratio was almost similar both for $\mathrm{Z}$ and $\mathrm{ZR}$ and higher ratio were found under low temperature than the other two treatments both in $\mathrm{Z}$ and $\mathrm{ZR}$ throughout the endosperm developing period except 7 DAA, which indicated that both $\mathrm{Z}$ and $\mathrm{ZR}$ play similar role on starch biosynthesis and the low temperature has positive effect on the reduction of chalkiness.

Table 1. Ratio of phytohormones content between rice grain and flag leaf

\begin{tabular}{|c|c|c|c|c|c|c|}
\hline \multirow[t]{2}{*}{$\begin{array}{c}\text { Name of } \\
\text { phytohormones }\end{array}$} & \multirow[t]{2}{*}{ Treatment } & \multicolumn{5}{|c|}{$\begin{array}{l}\text { Phytohormones ratio between grain and flag leaf (Mean of } \\
\text { 3 replications) }\end{array}$} \\
\hline & & 7 DAA & 14 DAA & $21 \mathrm{DAA}$ & $28 \mathrm{DAA}$ & $35 \mathrm{DAA}$ \\
\hline \multirow{3}{*}{ IAA } & low & 15.32 & 14.72 & 19.12 & 13.56 & 12.38 \\
\hline & High & 15.29 & 17.42 & 13.70 & 10.69 & 8.56 \\
\hline & Control & 17.91 & 17.07 & 16.82 & 11.17 & 9.41 \\
\hline \multirow{3}{*}{$\mathrm{GA}_{1}$} & low & 2.99 & 4.28 & 2.97 & 2.62 & 2.42 \\
\hline & High & 3.12 & 3.77 & 2.42 & 2.03 & 1.22 \\
\hline & Control & 3.13 & 3.81 & 2.45 & 2.34 & 1.83 \\
\hline \multirow{3}{*}{$\mathrm{GA}_{4}$} & low & 6.49 & 8.32 & 7.26 & 4.12 & 2.75 \\
\hline & High & 6.68 & 5.94 & 4.54 & 3.29 & 3.67 \\
\hline & Control & 7.00 & 8.11 & 6.23 & 3.82 & 3.88 \\
\hline \multirow{3}{*}{$\mathrm{Z}$} & low & 4.73 & 6.95 & 5.70 & 5.89 & 4.31 \\
\hline & High & 6.00 & 6.44 & 4.02 & 4.28 & 3.41 \\
\hline & Control & 5.26 & 6.87 & 3.76 & 3.44 & 3.15 \\
\hline \multirow{3}{*}{$\mathrm{ZR}$} & low & 4.31 & 7.03 & 6.87 & 4.60 & 3.08 \\
\hline & High & 6.02 & 5.42 & 3.41 & 3.07 & 2.42 \\
\hline & Control & 5.17 & 6.42 & 4.60 & 3.64 & 3.78 \\
\hline \multirow{3}{*}{$\mathrm{ABA}$} & low & 3.24 & 7.42 & 11.63 & 11.81 & 10.10 \\
\hline & High & 7.85 & 11.90 & 12.11 & 9.45 & 2.87 \\
\hline & Control & 5.21 & 11.13 & 12.14 & 9.81 & 5.40 \\
\hline
\end{tabular}

$\mathrm{IAA}=$ Indole-3-acetic acid, $\mathrm{GA}_{1}=$ Gibberellin $\mathrm{A}_{1}, \mathrm{GA}_{4}=$ Gibberellin $\mathrm{A}_{4}, \mathrm{Z}=$ Zeatin,

$\mathrm{ZR}=$ Zeatin riboside and $\mathrm{ABA}=\mathrm{Abscisic}$ acid, $\mathrm{DAA}=$ Days after anthesis

\section{Correlation between phyhormones and chalkiness}

The correlations among IAA, ABA, GAs $\left(\mathrm{GA}_{1}\right.$ and $\left.\mathrm{GA}_{4}\right), \mathrm{Z} \& \mathrm{ZR}$ and chalkiness were analyzed and the results are presented in Table 2 . It was found that IAA content in rice endosperm was not significantly correlated with chalkiness at 7 
and 14 DAA, while significant negative correlation was observed at 21, 28 and 35 DAA. A significant negative correlation was observed between chalkiness and both $\mathrm{GA}_{1}$ and $\mathrm{GA}_{4}$ throughout the grain filling period except $\mathrm{GA}_{1}$ at $28 \mathrm{DAA}$. All correlation coefficients between the $\mathrm{Z}$ and $\mathrm{ZR}$ level and chalkiness were negative. At 7 DAA the relationship between the $\mathrm{Z}$ and ZR level and chalkiness was weaker and insignificant, but in the later DAAs significantly negative correlations except for $\mathrm{ZR}$ at $35 \mathrm{DAA}$. The ABA levels in rice grains were significantly and positively correlated with chalkiness at 7, 14 and 21 DAA, but significantly and negatively correlated with chalkiness at later grain filling period (28 and 35 DAA).

\section{B. Discussion}

Grain filling is the most important developmental process that influences chalkiness, other quality indexes and grain yield of rice. The result showed that chalkiness was reduced by $52.75 \%$ under low temperature and increased by $27.82 \%$ under high temperature over the control. On the other hand, head rice was increased by $24.88 \%$ under low temperature and decreased by $14.33 \%$ under high temperature over the control. Furthermore, low temperature at grain developing stage is important for more economic returns for farmers due to reducing grain breakage or increasing head rice production (Koutroubas et al., 2004).

Table 2. Coefficient of correlation between chalkiness $(\%)$ and phytohormones level in the different DAAs

\begin{tabular}{llllll}
\hline $\begin{array}{c}\text { Name of } \\
\text { phytohormones }\end{array}$ & \multicolumn{1}{c}{$7 \mathrm{DAA}$} & \multicolumn{1}{c}{$14 \mathrm{DAA}$} & $21 \mathrm{DAA}$ & $28 \mathrm{DAA}$ & 35 DAA \\
\hline IAA & $0.5302^{\mathrm{NS}}$ & $-0.3938^{\mathrm{NS}}$ & $-0.8814^{* *}$ & $-0.7839^{*}$ & $-0.6751^{*}$ \\
$\mathrm{GA}_{1}$ & $-0.8753^{* *}$ & $-0.7928^{*}$ & $-0.6744^{*}$ & $-0.3435^{\mathrm{NS}}$ & $-0.7909^{*}$ \\
$\mathrm{GA}_{4}$ & $-0.8661^{* *}$ & $-0.8092^{* *}$ & $-0.7183^{*}$ & $-0.7188^{*}$ & $-0.6738^{*}$ \\
$\mathrm{Z}$ & $-0.4102^{\mathrm{NS}}$ & $-0.8105^{* *}$ & $-0.8492^{* *}$ & $-0.7990^{*}$ & $-0.7254^{*}$ \\
$\mathrm{ZR}$ & $-0.4474^{\mathrm{NS}}$ & $-0.8368^{* *}$ & $-0.8550^{* *}$ & $-0.7515^{*}$ & $-0.6347^{\mathrm{NS}}$ \\
$\mathrm{ABA}$ & $0.9017^{* *}$ & $0.9449^{* *}$ & $0.8072^{* *}$ & $-0.8386^{* *}$ & $-0.9629^{* *}$ \\
\hline
\end{tabular}

Note: $* *, *$ and NS indicate significant differences at $1 \%, 5 \%$ probability level, and nonsignificant, respectively

$\mathrm{IAA}=$ Indole-3-acetic acid, $\mathrm{GA}_{1}=$ Gibberellin $\mathrm{A}_{1}, \mathrm{GA}_{4}=$ Gibberellin $\mathrm{A}_{4}, \mathrm{Z}=$ Zeatin, $\mathrm{ZR}=$ Zeatin riboside and $\mathrm{ABA}=$ Abscisic acid, $\mathrm{DAA}=$ Days after anthesis

IAA is the most abundant endogenous hormones in plants, which regulates shoot growth, root branching, flowering and fruit ripening (Srivastava, 2002). It was found that low temperature treatment has an obvious impact to maintain higher IAA both in rice flag leaf and endosperm from the middle to the late grain filling stage. Early senescence of flag leaf under high temperature treatment might be due to the lower IAA content in flag leaf. IAA content in rice endosperm was 
found 19.12 times higher than in flag leaf. The positive effect of IAA on the photo-assimilate translocation within developing wheat grains have been reported by Darussalam Cole and Patrick (1998).

GAs control a wide range of processes during plant development, including seed germination, organ elongation and flower development (Yamaguchi, 2008). The result showed that lowering temperature increases $\mathrm{GA}_{1}$ and $\mathrm{GA}_{4}$ content both in rice flag leaf and endosperm throughout the grain filling period. It is well known that GAs levels are prevalent in developing grains, hence may be related to dry matter accumulation in grains. The relationship between chalkiness and GAs $\left(\mathrm{GA}_{1}\right.$ and $\left.\mathrm{GA}_{4}\right)$ was stronger and more negative at a significant level at 7 and 14 DAA than at 21, 28 and 35 DAA, which indicates that GAs $\left(\mathrm{GA}_{1}\right.$ and $\left.\mathrm{GA}_{4}\right)$ play an important role in the early to the middle grain filling stage than in the later stage for reduction of chalkiness.

Cytokinins are involved in the regulation of nutrient mobilization, senescence and flowering (Silverman et al., 1998). The contents of Z and ZR both in rice flag leaf and endosperm were significantly decreased irrespective of the treatments, but the lower temperature maintained a lead over high temperature and control treatments from 7 DAA to 35 DAA. Higher contents of $\mathrm{Z}$ and ZR in the flag leaf under low temperature causes delayed senescence of flag leaf. The results also showed that irrespective of the treatments, the highest levels of $Z$ and ZR were found at the early stage of grain development. The results also showed that high temperature stress reduced kernel $\mathrm{Z}$ and $\mathrm{ZR}$ contents as compared to control. Significant and negative correlation was observed between $Z \& Z R$, and chalkiness at the middle and late grain filling stage, which indicates that $\mathrm{Z}$ and ZR has a positive role in reduction of chalkiness at the middle and late grain filling stage. Considering the physiological function of cytokinines, the reduced cytokinin level resulted in depressed sink activity with higher chalkiness under high temperature stress.

ABA can promote dry matter accumulation in the sink organ which correlated with the growth rate of fruits or seeds (Wang et al., 1998; Yang et al., 2001). The result showed that relative to the control, high temperature stress increased ABA content both in rice flag leaf and endosperm from 7 DAA to 21 DAA but decreased ABA content at the later grain filling stage (28 and 35 DAA). A reverse trend was observed for low temperature treatment. Therefore, higher ABA content at the early grain filling stage enhances the senescence of flag leaves under high temperature stress. Rice grains mature earlier under high temperature than low temperature because $\mathrm{ABA}$ level plays an important role in positively regulating assimilate accumulation and accelerating maturation in developing grain (Yang et al., 1999; Duan et al., 1999). The relationship between ABA and chalkiness was significantly positive up to 21 DAA, but negative at 28 and 35 DAA. Therefore, rapid accumulation of ABA from the early to the midlate grain filling stage in rice endosperm, mediated by high temperature stress, 
enhanced chalkiness, but slower accumulation by low temperature from the early to the mid-late grain filling stage promoted lower chalkiness.

Among those phytohormones, IAA and ABA contents were much higher than $\mathrm{GA}_{1}, \mathrm{GA}_{4}, \mathrm{Z}$ and $\mathrm{ZR}$ in rice endosperm than in flag leaves which might reveal that IAA and ABA play important role for efficient production of starch as well as chalkiness in rice endosperm.

\section{Conclusion}

High temperature stress accelerates senescence of flag leaves, shortens grain filling period and accelerates poor grain filling or formation. In addition, high temperature also increases grain chalkiness and reduces head rice rate. The reduction of rice endosperm chalkiness was closely associated with the increased levels of IAA, $\mathrm{GA}_{1}, \mathrm{GA}_{4}, \mathrm{Z}, \mathrm{ZR}$ throughout the grain filling period and the increased level of ABA at $28 \& 35$ DAA under low temperature. The changes of endogenous phytohormone levels under different temperature regimes affected chalkiness through influencing the starch synthesis enzymes activity and other process. Rice grain chalkiness could be reduced by the regulation of phytohormone levels as well as by their balance in rice plants or grain either by using chemical, synthetic phytohormone or genetic improvement.

\section{Acknowledgements}

This work was financially supported by the National Natural Science Foundation of China (Grant No. NSFC 91317312 and NSFC 31570372) and National Agricultural Technology Program (NATP) phase-1, BARC, Dhaka, Bangladesh.

\section{References}

Borrell, A.M., A. Garside and S. Fukai. 1997. Improving efficiency of water use for irrigated rice in a semi-arid tropical environment. Field Crops Res. 52: 231-248.

Cao, X., Q. Zhu and J. Yang. 1992. Classification of source-sink types in rice varieties with corresponding cultivated ways. In: Xiong Z. and Min S. (eds), Prospects of Rice Farming for 2000, Zhejiang Sci. \& Tech. Press, Hangzhou. Pp. 361-372.

Chen, D.S., P. Cheng, D.H. Li and L.T. Xiao. 2011. Studies on measurement system for rice chalkiness based on computer image processing. J. of Hunan Agril. Univ. (Natural Sciences). 37: 469-473.

Darussalam Cole, M.A. and J.W. Patrick. 1998. Auxin control of photoassimilate transport to and within developing grains of wheat. Australian J. of Pl. Physiology. 25: 69-77.

Dong, H., D.Z. Sang, P. Wang and J.C. Yang. 2006. Difference in chalky character of the grains at different positions within a rice panicle. Acta Agronomica Sinica. 32: 103-111.

Duan, J., C.E. Tian, C.Y. Liang, Y.W. Huang and H.X. Liu. 1999. Dynamic changes of endogenous plant hormones in rice grains in different parts of panicle at grain filling stage. Acta Botanical Sinica. 41(1): 75-79 (in Chinese) 
Gummert, M. 2010. Measuring white rice quality. Postharvest fact sheet of rice knowledge bank. International Rice Research Institute (IRRI), Los Baños, Philippines.

Han, Y., M. Xu, X. Liu, C. Yan, S.S. Korban, X. Chen and M. Gu. 2004. Genes coding for starch branching enzymes are major contributors to starch viscosity characteristics in waxy rice (Oryza sativa L.). Plant Sci. 166: 357-364.

Koutroubas, S.D., F. Mazzini, B. Pons and D.A. Ntanos. 2004. Grain quality variation and relationships with morpho-physiological traits in rice (Oryza sativa L.) genetic resources in Europe. Field Crops Res. 86: 115-130.

Naik, P.K. and P.K. Mohapatra. 1999. Ethylene inhibitors promote male gametophyte survival in rice. Plant Growth Regulation. 28: 29-39.

Patindol, J. and Y.J. Wang. 2003. Fine structures and physicochemical properties of starches from chalky and translucent rice kernels. J. Agril \& Food Chem. 51: 2777-2784.

Silverman, F.P., A.A. Assiamah and D.S. Bush. 1998. Membrane transport and cytokinin action in root hairs of Medicago sativa. Planta. 205: 23-31.

Srivastava, L.M. 2002. Plant growth and development: Hormones and Environment. Academic Press, San Diego, California, USA.

Wang, Z., J. Yang, Q. Zhu, Z. Zhang, Y. Lang and X. Wang. 1998. Reasons for poor grain plumpness in inter-subspecific hybrid rice. Acta Agronomica Sinica. 24(6): 782-787.

Wobus, U. and H. Weber. 1999. Seed maturation: genetic programmes and control signals. Current Opinion on Plant Biology. 2: 33-38.

Yamaguchi, S. 2008. Gibberellin metabolism and its regulation. Annual Review of Plant Biology. 59: 225-51.

Yang, J.C., Z.Q. Wang, Q.S. Zhu and B.L. Su. 1999. Regulation of ABA and GA to the grain filling of rice. Acta Agronomica Sinica. 25(3): 341-348 (in Chinese).

Yang, J., S. Peng, R.M. Visperas, A.L. Sanico, Q. Zhu and S. Gu. 2000. Grain filling pattern and cytokinin content in the grains and roots of rice plants. Plant Growth Regulation. 30: 261-270.

Yang, J., J. Zhang, Z. Wang, Q. Zhu and W. Wang. 2001. Hormonal changes in the grains of rice subjected to water stress during grain filling. Plant Physiology. 127: 315-323.

Yoshida S, Forno DA, Cock JH, Gomez KA. 1976. Laboratory manual for physiological studies of rice. $3^{\text {rd }}$ ed. Philippines: International Rice Research Institute.

Zhang, H., S. Zhang, J. Yang, J. Zhang and Z. Wang. 2008. Postanthesis moderate wetting drying improves both quality and quantity of rice yield. Agronomy J. 100: 726-734. 
